\title{
Mucosal-Associated Invariant T Cells: New Insights into Antigen Recognition and Activation
}

\author{
Xingxing Xiao ${ }^{1,2}$ and Jianping Cai ${ }^{1,2 *}$ \\ ${ }^{1}$ State Key Laboratory of Veterinary Etiological Biology, Key Laboratory of Veterinary Parasitology of Gansu Province, \\ Lanzhou Veterinary Research Institute, Chinese Academy of Agricultural Sciences, Lanzhou, China, ${ }^{2}$ Jiangsu Co-Innovation \\ Center for Prevention and Control of Animal Infectious Diseases and Zoonoses, Yangzhou, China
}

OPEN ACCESS

Edited by:

Luc Van Kaer, Vanderbilt University,

United States

Reviewed by:

S. M. Mansour Haeryfar,

University of Western

Ontario, Canada

Derek G. Doherty,

Trinity College, Dublin, Ireland

*Correspondence: Jianping Cai

caijianping@caas.cn

Specialty section:

This article was submitted

to T Cell Biology,

a section of the journal

Frontiers in Immunology

Received: 16 September 2017

Accepted: 30 October 2017

Published: 10 November 2017

Citation:

Xiao X and Cai J (2017) Mucosal-

Associated Invariant T Cells:

New Insights into Antigen

Recognition and Activation.

Front. Immunol. 8:1540.

doi: 10.3389/fimmu.2017.01540
Mucosal-associated invariant T (MAIT) cells, a novel subpopulation of innate-like T cells that express an invariant T cell receptor (TCR) $\alpha$ chain and a diverse TCR $\beta$ chain, can recognize a distinct set of small molecules, vitamin $B$ metabolites, derived from some bacteria, fungi but not viruses, in the context of an evolutionarily conserved major histocompatibility complex-related molecule 1 (MR1). This implies that MAIT cells may play unique and important roles in host immunity. Although viral antigens are not recognized by this limited TCR repertoire, MAIT cells are known to be activated in a TCR-independent mechanism during some viral infections, such as hepatitis $C$ virus and influenza virus. In this article, we will review recent works in MAIT cell antigen recognition, activation and the role MAIT cells may play in the process of bacterial and viral infections and pathogenesis of non-infectious diseases.

Keywords: mucosal-associated invariant T cells, antigens, recognition, activation, diseases

\section{INTRODUCTION}

$\mathrm{T}$ lymphocyte activation is best explained by conventional $\mathrm{T}$ cells using a very diverse repertoire of T cell receptors (TCRs), which recognize various antigenic peptides presented in the context of classical major histocompatibility complex (MHC) class I or class II molecules $(1,2)$. This response is adaptive or acquired and is different from the innate immune response, which is MHC and TCR independent. In humans and mice, however, there are also other important groups of $\mathrm{T}$ cells, known as non-conventional $\mathrm{T}$ cells, which can be activated in a mechanism distinct from conventional $\mathrm{T}$ cells. Unlike conventional $\mathrm{T}$ cells, which recognize peptide antigens, invariant natural killer $\mathrm{T}$ (iNKT) cells and mucosal-associated invariant $\mathrm{T}$ (MAIT) cells recognize lipids and vitamin B metabolites, respectively $(3,4)$. Once activated in a TCR-dependent and/or -independent manner, these unconventional $\mathrm{T}$ cells produce cytokines and some cytotoxic effector molecules, such as tumor necrosis factors (TNFs), perforin, and granzymes (Gzms) (5-8). Because these cells share properties of both the adaptive and innate responses, they are termed innate-like $\mathrm{T}$ cells, which express TCR with limited diversity and respond rapidly to relatively conserved antigen challenge, and can be said to represent a bridge between the adaptive and innate immune systems $(8-11)$.

Like the intensively studied innate-like T cells, iNKT cells are restricted by CD1d and express a semi-invariant TCR V $\alpha 24-J \alpha 18$ in humans and V $\alpha 14-J \alpha 18$ in mice with a biased repertoire of V $\beta$ chains $(6,12-15)$. In mice, iNKT cells are abundant in the liver, bone marrow and adipose tissue, but less in other tissues, such as spleen, blood and lung (16-18); however, in humans, they are rare in blood 
and liver compared to mice (19). Nevertheless, a recent study shows that iNKT cells are abundant in human omentum (20). iNKT cells recognize synthetic, self, and microbial lipid-based antigens presented by CD1d $(6,21,22)$. Upon TCR or cytokinemediated activation, iNKT cells rapidly produce cytokines and chemokines, exert cytotoxic activity by high expression of Gzms, perforin, and FasL, and regulate other immune cells $(6,23)$. As such, iNKT cells play important roles in combating infectious diseases and are also implicated in host responses to autoimmune diseases, chronic inflammatory diseases, and cancers $(17,24)$.

Mucosal-associated invariant $\mathrm{T}$ cells, a new subpopulation of innate-like T cells, have many similarities to iNKT cells, both in immunological properties and functions. Both cell types express semi-invariant TCRs, are restricted by monomorphic molecules, and can rapidly produce proinflammatory cytokines. With an increasing understanding of the antigens recognized by MAIT cells and their activation, the roles MAIT cells play in the immune response have been gradually revealed. Here, this review will summarize the discovery and phenotypic characterization of MAIT cells, then focus on MAIT cell antigen recognition and activation, and finally, discuss the roles of MAIT cells in infectious and non-infectious diseases.

\section{MAIT CELL DISCOVERY AND PHENOTYPE}

In 1993, Porcelli et al. found that peripheral blood $\alpha \beta \mathrm{CD}^{-} \mathrm{CD}^{-}$ [double negative (DN)] T cells from healthy people preferentially expressed two invariant TCR $\alpha$ chains (15). One invariant TCR $\alpha$ chain, comprised of the V $\alpha 24$ and J $\alpha 18$ gene segments (V $\alpha 14$ and Jo18 in mice), was expressed by what we now know as iNKT cells, while the other invariant TCR $\alpha$ chain used the V $\alpha 7.2$ and $J \alpha 33$ gene segments (V $\alpha 19$ and $J \alpha 33$ in mice) $(12,14,15)$. Then, in 1999, a seminal study by Tilloy et al. showed that this invariant TCR $\alpha \mathrm{V} \alpha 7.2 / \mathrm{V} \alpha 19-\mathrm{J} \alpha 33$ chain was expressed by a new type of $\mathrm{T}$ cell subset present in humans, mice, and cattle, indicating conservation between mammalian species (25). In 2003, Treiner et al. observed that this novel subset of $\mathrm{T}$ cells was preferentially located in the intestinal lamina propria (LP) of humans and mice, and therefore, named these cells "mucosalassociated invariant T (MAIT) cells" (26). They also showed that MAIT cells were restricted by MR1, a monomorphic class I-related MHC molecule that is highly conserved in mammalian species $(26,27)$.

Although originally described as $\alpha \beta$ DN T cells, MAIT cells, which are $\mathrm{CD}^{+}$, may also be $\mathrm{CD} 4^{+}$and/or $\mathrm{CD}^{+}(25,28,29)$. In humans, only $2-11 \%$ of MAIT cells in blood express CD4, while in V $\alpha 19 \mathrm{iTg}-\mathrm{C}^{-/-} \mathrm{C} 57 \mathrm{BL} / 6$ (B6) mice, up to $50 \%$ of splenic MAIT cells are $\mathrm{CD}^{+}$, implying different coreceptor requirements between humans and transgenic ( $\mathrm{Tg}$ ) mice (29). In wild-type (WT) mice (B6 or BALB/c), MAIT cells also consist of three subsets $\left(\mathrm{DN}, \mathrm{CD}^{+}\right.$, and $\left.\mathrm{CD} 8^{+}\right)$, and their frequencies vary in different tissues and strains (28). CD8 ${ }^{+}$MAIT cells are present in different frequencies in humans and mice and are a mixture of CD8 $\alpha \alpha$ and CD8 $\alpha \beta$ cells (28-31). Contrary to iNKT cells, MAIT cells are abundant in humans, but rare in mice $(17,32)$. Moreover, as with iNKT cells, MAIT cells also display a restricted TCR repertoire. In humans, the majority of MAIT cells express a
TCR comprised of a canonical V $\alpha 7.2-\mathrm{J} \alpha 33$ TCR $\alpha$ rearrangement paired with limited TCR $\beta$ chains (predominantly $\mathrm{V} \beta 2$ or $\mathrm{V} \beta 13$ ), and less frequent usage of the non-canonical V $\alpha 7.2-\mathrm{J} \alpha 12 / \mathrm{J} \alpha 20$ TCR $\alpha$ rearrangement $(15,25,29,33)$. Mouse MAIT cell TCRs utilize an invariant V $\alpha 19-\mathrm{J} \alpha 33 \mathrm{TCR} \alpha$ chain predominantly paired with a $\mathrm{V} \beta 6$ or $\mathrm{V} \beta 8$ chain $(25,28)$. The variability of MAIT TCR $\beta$ chains may contribute to the functional heterogeneity of MAIT cells as immune effectors against various microbes (34).

Mucosal-associated invariant $\mathrm{T}$ cells express several cell surface proteins that can be used for identification and characterization (Table 1). Both human and mouse MAIT cells have a memory phenotype and may express transcription factors promyelocytic leukemia zinc finger (PLZF), retinoic acid-related orphan receptor $\gamma \mathrm{t}(\mathrm{ROR} \gamma \mathrm{t})$, and T-bet $(28,35-37)$. Detection of other cell surface proteins in various combinations has been used to detect MAIT cells. Both human and mouse MAIT cells express homing receptors, such as CXCR6, CCR9, $\alpha 4 \beta 7$, and/or CD103, which are consistent with their ability to migrate to the skin, liver, lung, and gut LP $(28,30)$. MAIT cells also express cytokine receptors, such as IL-7R $\alpha$, IL-12R, and IL-18R $\alpha(28,38-40)$.

Initially, the reagent used to specifically identify and study human MAIT cells $\left(\mathrm{CD} 3{ }^{+} \mathrm{CD} 161^{\mathrm{hi}} \mathrm{V} \alpha 7.2^{+} \mathrm{T}\right.$ cells) was an antibody mix including anti-V $\alpha 7.2$ and anti-CD161 mAb (35); however, because V $\alpha 7.2$ TCR is also expressed by conventional T cells and CD1b-restricted germline-encoded, mycolyl lipid-reactive (GEM) T cells (V $\alpha 7.2-\mathrm{J} \alpha 9)(46,47)$, an anti- $\mathrm{V} \alpha 7.2 \mathrm{mAb}$ alone is no longer considered an appropriate tool to study MAIT cells in humans. MAIT cells are phenotypically CD161 ${ }^{\text {hi }}$ but CD161 is downregulated on MAIT cells in HIV patients (48), so CD161 is not a useful marker when examining specimens from HIV positive patients and potentially other cases. Because MAIT cells are rare in mice and there is no anti-V $\alpha 19$ specific $\mathrm{mAb}$ available, researchers generated $\mathrm{B} 6 \mathrm{Tg}$ mice expressing only the TCR Vo19-J $\alpha 33$ (V $\alpha 19$ i) chain to study MAIT cell development, phenotype, and antigen specificity $(35,41,49)$. Unfortunately, maybe because of the developmental differences in MAIT cells between species, MAIT cells in $\mathrm{Tg}$ mice do not reflect the properties of MAIT cells in WT mice or humans $(28,41,43)$. Recently, researchers have developed two new tools to study human and mouse MAIT cells: MR1-antigen tetramers $(29,50)$ and the B6-MAIT ${ }^{\text {CAST }}$ mouse, whose augmented MAIT cells are phenotypically very similar to human and WT mouse MAIT cells (Table 1) (38). These two new tools have facilitated the characterization of "natural" mouse MAIT cells, and the use of MR1-antigen tetramers circumvents the overlap/limitations of surrogate phenotypes to define MAIT cells in humans.

\section{MAIT CELL ANTIGENS}

Mucosal-associated invariant $\mathrm{T}$ cells are known as nonconventional $\mathrm{T}$ cells in part because they recognize non-peptide antigens presented by the non-polymorphic MR1 molecule (51). MR1 has a standard MHC-I fold, and the antigen-presenting process of MR1 is similar to MHC-I and CD1d molecules, leading Huang et al. to speculate that MR1 could present peptide or lipid antigens to MAIT cells (52). Kjer-Nielsen et al. later showed 
TABLE 1 | Surface phenotype of MAIT cells in mouse and human.

\begin{tabular}{|c|c|c|c|c|}
\hline & \multicolumn{4}{|c|}{ MAIT cells } \\
\hline & Vo19iTg-C $\alpha^{-/-}$B6 mouse & WT B6 mouse & B6-MAIT ${ }^{\text {CAST }}$ mouse & Human \\
\hline TCR $\alpha$ chain & V $\alpha 19-J \alpha 33(25,41)$ & V $\alpha 19-J \alpha 33$ (25) & V $\alpha 19-J \alpha 33(25,38)$ & V $\alpha 7.2-J \alpha 33 / 20 / 12(25,29)$ \\
\hline TCR $\beta$ chain & $\begin{array}{l}\text { Predominantly } \vee \beta 8.1 / 8.2 / 6 \\
(25,41)\end{array}$ & Predominantly V $\beta 8.1 / 8.2 / 6(25,28)$ & $\begin{array}{l}\text { Predominantly } \\
\text { V } \beta 8.1 / 8.2 / 6(25,38)\end{array}$ & Predominantly V $\beta 2 / 13(25,29)$ \\
\hline Coreceptor & CD8 ${ }^{\circ}, \mathrm{DN}, \mathrm{CD} 4(29)$ & CD8, DN, CD4 $(26,28)$ & $\mathrm{CD} 8^{\circ}, \mathrm{DN}(38)$ & $\mathrm{CD} 8 \alpha \alpha, \mathrm{CD} 8 \alpha \beta^{\mathrm{lo}}, \mathrm{DN}, \mathrm{CD} 4^{\mathrm{lo}}(29)$ \\
\hline $\begin{array}{l}\text { Egress from the thymus } \\
\text { as }\end{array}$ & Naive $(35,41)$ & Naive (42) & n.d. & Naive $(35,42)$ \\
\hline Phenotype in periphery & Naive $(35,41)$ & Effector memory $(28,36)$ & Memory (38) & Effector memory $(35,36)$ \\
\hline Transcription factor & PLZFº (35) & PLZF, ROR $\gamma t^{\text {thi }}$, T-bet $^{\text {lo }}(28,36,37)$ & PLZF, RORyt (38) & PLZF, RORyt, T-bet $(35,36)$ \\
\hline Activation marker & $\begin{array}{l}\text { CD25, CD69, NK1.1 (11, } \\
41,43)\end{array}$ & CD25, CD69'o, NK1.1' $(28,37,38)$ & CD25, CD69 (38) & CD25, CD69, CD161 hi $(11,35,39,44)$ \\
\hline Differentiation marker & $\mathrm{CD} 44^{\circ \circ} \mathrm{CD} 122^{\mathrm{lo}}(35)$ & $\mathrm{CD}_{4}{ }^{\text {hi }} \mathrm{CD} 6 \mathrm{~L}^{\text {lo }}(28)$ & CD44hi $(38)$ & $\mathrm{CD}{ }^{\mathrm{hi}} \mathrm{CD} 2 \mathrm{~L}^{\text {lo }} \mathrm{CD} 45 \mathrm{RO}^{+} \mathrm{CD} 27^{+}(44)$ \\
\hline Homing receptor & CXCR3, $\alpha 4 \beta 1$ (43) & CXCR6, CCR6, CCR9, CD103 (28) & $\begin{array}{l}\text { CD103, CXCR6, } \\
\text { CCR6 (38) }\end{array}$ & $\begin{array}{l}\text { CCR2, CCR5, CCR6, CXCR6, CCR9, } \alpha 4 \beta 7 \text {, } \\
\text { CD103 }(26,30)\end{array}$ \\
\hline Cytokine receptor & n.d. & IL-7R $\alpha, \operatorname{IL}-18 R \alpha$, IL-12R $\beta 1(28,38)$ & $\begin{array}{l}\text { IL-7R } \alpha, \text { IL-18R } \alpha \\
\text { IL-12R } \beta 1 \text { (38) }\end{array}$ & IL-7R $\alpha$, IL-12R, IL-18R $\alpha$, IL-23R $(30,40,45)$ \\
\hline
\end{tabular}

n.d., not described.

that MAIT cells could recognize small molecule metabolites, including reduced 6-hydroxymethyl-8-D-ribityllumazine (rRL6- $\mathrm{CH}_{2} \mathrm{OH}$ ), 7-hydroxy-6-methyl-8-D-ribityllumazine (RL-6-Me7-OH), and 6,7-dimethyl-8-D-ribityllumazine (RL-6,7-diMe), derived from the riboflavin [vitamin B2 (VB2)] biosynthetic pathway of bacteria (4). These compounds all have a bicyclic structure and a ribityl tail, but differ in their potency for activating MAIT cells (Table 2). Further research found that the most potent agonists were unstable pyrimidine intermediates, including 5-(2-oxoethylideneamino)-6-D-ribitylaminouracil (5-OERU) and 5-(2-oxopropylideneamino)-6-D-ribitylaminouracil (5-OP-RU), which are also derived from the VB2 biosynthetic pathway and are formed by the spontaneous non-enzymatic reactions between 5-amino-6-D-ribitylaminouracil (5-A-RU) and glyoxal or methylglyoxal, respectively (50). Although these three pyrimidines have the same single-ring structure and ribityl tail, 5-A-RU does not stimulate MAIT cells because MR1 is not refolded efficiently with 5-A-RU alone $(50,53)$. Because 5-OE-RU and 5-OP-RU are very unstable in water, Mak et al. preformed 5-OP-RU in DMSO- $d_{6}$ and then diluted it in aqueous buffer, and as a result, improved its purity, quantity, and stability (54). They also designed and synthesized a new reagent $\mathbf{1 1}$, a very potent analogue of 5-OP-RU, which was completely stable in water and showed a similar functional profile as 5-OP-RU, making it a valuable antigen analogue that can be used for MAIT cell research. Presently, six distinct antigens have been identified that are capable of activating MAIT cells, five VB2 metabolites and 5-OP-RU analogue. Although similar in structure, these antigens differ in their abilities to activate MAIT cells (Table 2) $(4,50,54)$. In addition to these six agonists, MAIT cell inhibitors have also been identified, such as 6-formylpterin (6-FP) and acetyl-6-formylpterin (Ac-6-FP), which are folic acid [vitamin B9 (VB9)] derivatives with a bicyclic structure and a formyl group
$(4,53,55,56)$; Ac-6-FP is a stronger inhibitor than 6-FP, which is the first MR1 ligand described $(4,53)$.

Another reason MAIT cells are termed non-conventional is that they use a semi-invariant TCR and are restricted by a highly conserved MR1, implying a MAIT cell antigen repertoire with limited diversity (56). Known MAIT cell antigens only fill the $\mathrm{A}^{\prime}$ pocket of MR1 yet the heterogeneity of MAIT TCR chain $\mathrm{J} \alpha$ and V $\beta$ usage suggested that MAIT cells may recognize additional antigens $(17,29,30,56)$. Therefore, finding more antigens will be helpful to understand MAIT cell recognition and activation. For example, the five known VB2 metabolite antigens, which are only synthesized by plants as well as many bacteria and yeast $(56,58)$, may reflect a primitive mechanism of self/non-self discrimination $(59,60)$. Recently, Keller et al. found that aside from VB9 derivatives and VB2 precursors, MR1 also presents drugs and drug-related molecules with diverse chemical structures (57), indicating that the pocket of MR1 has sufficient plasticity to accommodate a variety of molecules, including agonists or inhibitors of MAIT cell activation, such as diclofenac (DCF), 5-hydroxy DCF (5-OH-DCF) and $4^{\prime}$-hydroxy DCF ( $4^{\prime}$-OH-DCF), and 3 -formylsalicylic acid (3-F-SA), 5-formylsalicylic acid (5-F-SA), 2-hydroxy-1-naphthaldehyde (2-OH-1-NA) and 2,4-diamino-6-formylpteridine (2,4-DA-6-FP), respectively. Therefore, we can modulate MAIT cell function by some of these drugs. Taken together, researchers have reported several agonists and inhibitors derived from VB metabolites and drugs or drug-related molecules. Of them, some molecules, such as DCF, DCF metabolites, and 5-FSA, only activate specific subsets of MAIT cells with specific TCR $\beta$ chain usage (57), suggesting that the use of MAIT TCR $\beta$ chains "fine tunes" the responsiveness to certain antigens. Indeed, the effector responses of MAIT cells against Escherichia coli (E. coli) and Candida albicans (C. albicans) exhibit TCR $\beta$ 
TABLE 2 | The role of MR1 ligands in activation of MAIT cells.

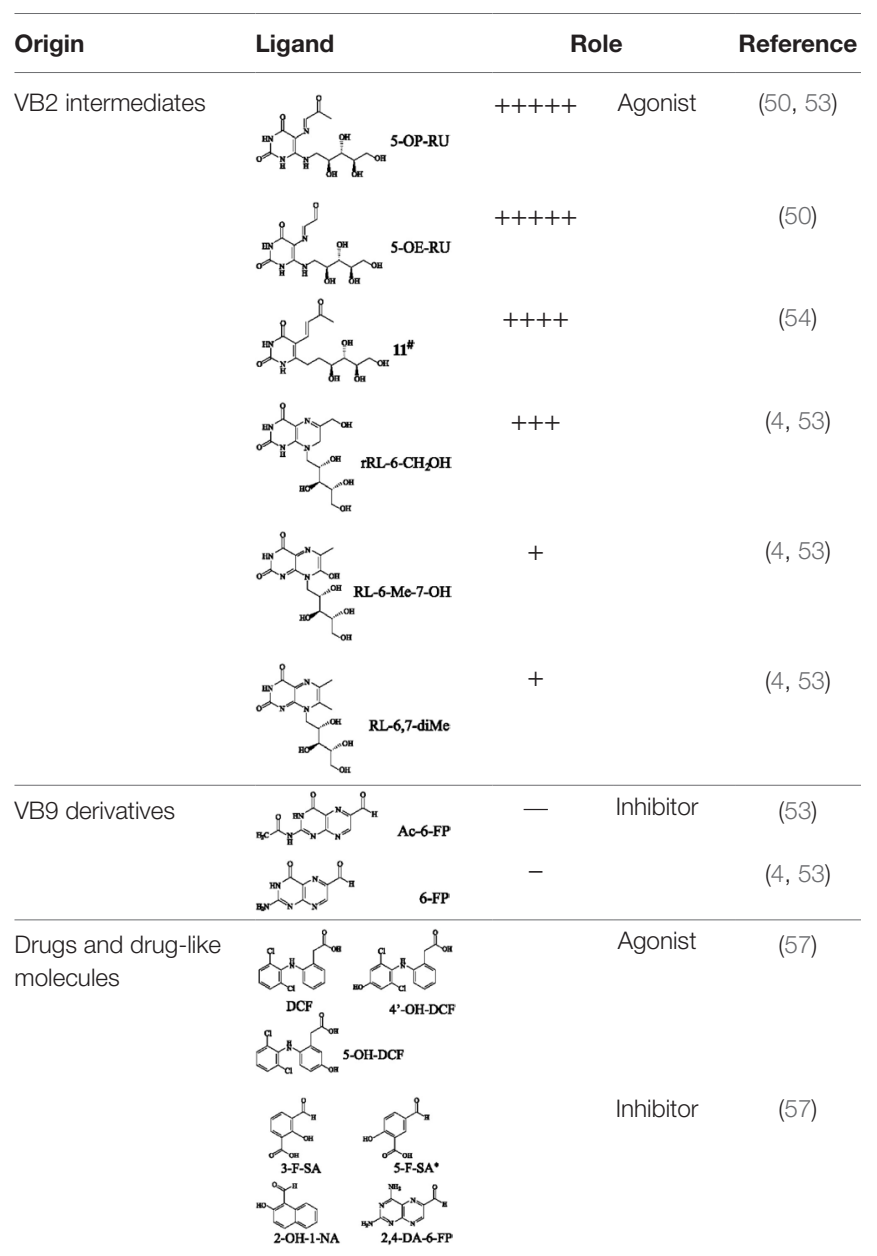

\#: a potent analogue of 5-OP-RU.

*: also as an agonist of activation of MAIT cells expressing a TRBV6-4.

+ : the potency for activating.

-: the potency for non-activating.

chain bias. MAIT cells that are hyporesponsive to E. coli infection express V $\beta 8, V \beta 13.1$, and V $\beta 13.6$, while MAIT cells that are responsive to $C$. albicans infection have a different TCR $\beta$ chain bias (34). To date, there are no reports about the nature of endogenous MAIT cell antigens; the antigens involved in MR1-mediated MAIT cell activation are all exogenous, which is inconsistent with iNKT cells $(6,24)$.

The identification of specific MAIT cell antigens has resulted in the generation of MR1-antigen tetramers. The first generation of tetramers was generated by loading of MR1 with rRL-6- $\mathrm{CH}_{2} \mathrm{OH}$ (29), which had lower affinity for staining MAIT cells. Now, however, the second generation of MR1 tetramers is prepared with 5-OP-RU, which is the most potent MAIT cell activator so far (50). Although MR1-antigen tetramers have facilitated studying and understanding mouse and human MAIT cell research, the use of MR1 tetramer staining has some limitations. Several authors have shown that MR1-antigen tetramer ${ }^{+}$ $\mathrm{T}$ cells are not all MAIT cells $(10,61)$ and contain $1-4 \%$ V $\alpha 7.2^{-} \mathrm{T}$ cells, one subset of which detects infection with the riboflavin auxotroph Streptococcus pyogenes (S. pyogenes) in a TCR-dependent manner (61), implying that MR1 can present different ligands to MR1-antigen tetramer ${ }^{+} \mathrm{T}$ cells depending on the TCR $\alpha$ chain usage. Indeed, Lepore et al. discovered a novel population of MR1-restricted T cells (MR1T), which are MR1antigen tetramer ${ }^{+} \mathrm{T}$ cells but are not MAIT cells, have variable TCR $\alpha$ chains, and can be activated by self-antigens (62). These antigens are purified from two tumor cell lines, and can activate MR1T cells without forming a Schiff base with MR1. However, the nature of self-antigens still need to be further studied.

\section{MAIT TCR-MR1-ANTIGEN INTERACTIONS}

Several reviews are available that discuss MAIT TCR-MR1antigen interactions $(30,51,56,59)$; therefore, we will focus on the difference between recently discovered antigens, drugs and drug-related metabolites, and riboflavin metabolites in interaction with TCR-MR1. Comparisons of the ternary structures of MAIT TCR-MR1-6-FP and MAIT TCR-MR1-RL-6-Me-7-OH suggested that MR1 has plasticity in its ability to accommodate ligands $(59,60)$. Consistent with this, there are more MR1 ligands discovered recently $(50,57)$. Whether it be VB2 metabolites or drugs and drug-related metabolites, the MAIT TCR docks with MR1-antigen complexes in an approximately orthogonal and central manner $(50,57,63)$. However, based on crystallography studies, DCF and 5-OH-DCF presentation is dramatically different from that of VB2 antigens, which are presented in the MR1-binding cleft with the plane of the central phenylacetic acid ring basically perpendicular to that of the phenylacetic acid ring of DCF when it interacts with MR1-TCR (57).

Crystal structures of the MR1-antigen-MAIT TCR complex revealed that Tyr95 $\alpha$ (Y95 $\alpha)$ on MAIT TCR $\alpha$ chain interacts with ribityl tail of VB2 derived antigens, which plays a vital role in activating MAIT cells $(29,53,63,64)$; however, Y95 $\alpha$ does not interact with DCF and 5-OH-DCF, which lack a ribityl group (57). DCF and 5-OH-DCF activate MAIT cells when the Y95 $\alpha$ aromatic ring piles against the phenylacetic acid ring forming van der Waals forces with E99 $\beta$ from TCR $\beta$ chain (57). In contrast, inhibitors, such as 3-F-SA, 2-OH-NA, and 2,4-DA-6-FP, do not directly interact with the MAIT TCR (57), which is also the case with VB9-derived inhibitors (53). Therefore, small organic molecules acting as MAIT cell antigens directly contact the MAIT TCR, and vice versa.

\section{MAIT CELL ACTIVATION}

Mucosal-associated invariant $\mathrm{T}$ cells are known to be activated by some bacteria and yeasts, but not by viruses, in an MR1dependent manner $(11,65)$. However, recent studies have shown that not only in viral diseases $(7,66)$, but also in some non-infectious diseases (67-69), MAIT cells can be activated in an MR1-independent manner. In either the MR1-dependent or -independent manner, upon activation, MAIT cells rapidly proliferate, secrete proinflammatory cytokines and other factors resulting in lysis of the infected cells, and have the capacity for B cell help $(5,70,71)$. 
Microbes which utilize the riboflavin biosynthetic pathway activate MAIT cells in an MR1-dependent manner (Figure 1A) $(11,37,65,72,73)$. Human MAIT TCRs recognize VB2-derived antigens presented by MR1, and MAIT cells then upregulate the expression of CD25, CD69, and CD161, secrete Th1-type cytokines (IFN- $\gamma$ and TNF- $\alpha$ ) and Th17-type cytokines (IL-17 and IL-22), but do not secrete Th2-type cytokines (30, 40, 74), which is consistent with their expression of transcription factors like T-bet and RORyt (36, 44, 75). Mouse MAIT cells secrete high levels of IL-17, but lower levels of IL-4, IL-10, IL-13, IFN- $\gamma$, TNF- $\alpha$, and GM-CSF upon activation with anti-CD3/ CD28-coated beads $(28,30,38)$. In addition to the secretion of proinflammatory cytokines, MAIT cells produce Gzms (GzmA, GzmK, and GzmB) and perforin, which function to effectively lyse infected cells $(5,76)$. Primary human MAIT cell activation is inefficient after in vitro stimulation with soluble ligands in an MR1-dependent manner, but also requires toll-like receptor (TLR) signaling and antigen-presenting cell (APC) activation (77). Consistent with this, accumulation and enrichment of MAIT cells in vivo not only requires VB2-derived antigens but also costimulatory signals, such as TLR agonists (37). Therefore, to establish a murine model of bacterial infection for MAIT cell studies, mice can be inoculated with synthetic antigens and TLR agonists such as CpG and poly I:C first, to promote MAIT cell accumulation and proliferation.

Like iNKT cells, MAIT cells can also be activated in an MR1independent manner (Figure 1B). Several studies have shown that MR1-independent MAIT cell activation is dependent on IL-18 in synergy with other inflammatory mediators $(7,45,66)$, which is consistent with the high expression of IL- $18 \mathrm{R} \alpha$ on MAIT cells $(30,60)$. Upon cytokine-mediated activation in viral infections, MAIT cells can produce IFN- $\gamma$ and GzmB, which

\section{A Antigen-mediated activation of MAIT cells B Cytokine-mediated activation of MAIT cells}
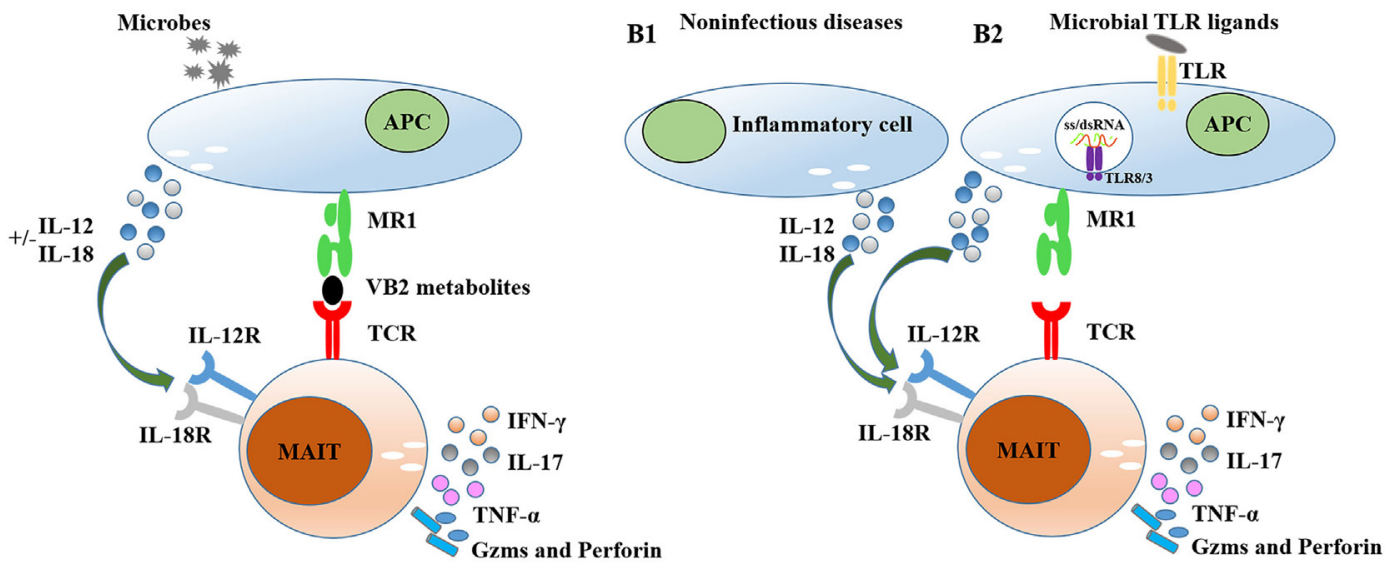

C SAg-triggered activation and anergy of MAIT cells

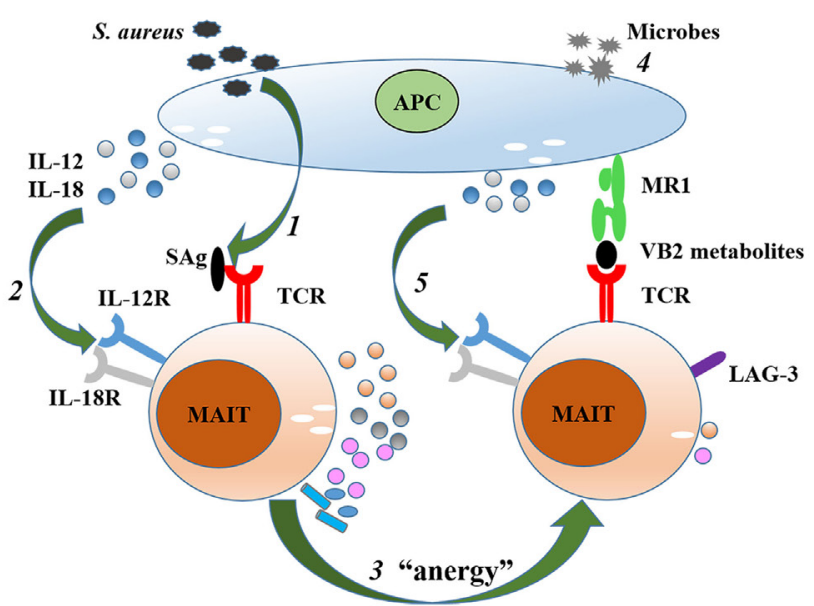

FIGURE 1 | Mechanisms of mucosal-associated invariant T (MAIT) cell activation. (A) MAIT cells are activated by microbes that utilize a riboflavin biosynthetic pathway in an MR1-dependent manner. This activation can be enhanced when infected cells produce IL-12 and IL-18. (B) MAIT cells are activated by cytokines (IL-12 and IL-18) in an MR1-independent manner. These cytokines can be produced by inflammatory cells in non-infectious diseases (B1) or infected cells in viral disease (B2). (C) MAIT cells are activated by superantigen (SAg) in a T cell receptor (TCR)B-dependent manner (1) and/or cytokine-mediated (2 and 5) pathway, then upregulate inhibitory receptors such as lymphocyte-activation gene 3 (LAG-3), and then are anergized (3) to subsequent bacterial challenge (4). 
can be suppressed or even abrogated by anti-IL-18 but not by MR1-blocking $(7,66,78)$. In contrast, MAIT cells are activated via different mechanisms in response to different viral infections. In dengue virus (DENV) infection, MAIT cells respond to both IL-12 and IL-18, while in hepatitis C virus (HCV) and influenza virus (IAV) infection, MAIT cell activation is predominantly dependent on IL-18, which is produced by CD14 ${ }^{+}$ monocytes $(66,75,78)$. Moreover, IFN- $\alpha / \beta$ may also play a role in MAIT cell activation during viral infection (7). Corresponding to activation of MAIT cells in viral infection, previous studies showed that TLR8 and TLR3 are potent activators of MAIT cells, promoting the secretion of IL- 12 and IL-18 by APCs $(7,45,79)$. In addition to viral infections, MAIT cells also respond to infections with some bacterial species, such as Mycobacterium tuberculosis, Mycobacterium bovis bacillus Calmette-Guérin, and Enterococcus faecalis, in an MR1-independent manner (45, 80, 81). So, although there are two pathways of MAIT cell activation in response to bacterial infections, MR1-dependent and MR1independent, the relative contribution of the two pathways is still unclear. Interestingly, MAIT cells are also activated in some non-infectious diseases. In patients with systemic lupus erythematosus (SLE), MAIT cells can be activated by IFN- $\alpha$, IL-15, and IL-12 plus IL-18 in the absence of exogenous antigens (69). The positive correlation between the plasma concentration of these cytokines and the expression levels of CD69 on MAIT cells suggests that proinflammatory cytokines may activate MAIT cells and may play a role in the pathogenesis of SLE and possibly other inflammatory processes.

Interestingly, recently a new study showed that, apart from MR1-dependent and cytokine-mediated activation, MAIT cells can also be activated by superantigens (SAgs) produced by Staphylococcus aureus and S. pyogenes in a TCR V $\beta$-dependent manner (Figure 1C) (82), following which Sandberg et al. wrote a commentary to highlight this new discovery (83). Moreover, SAgs also activate MAIT cells through IL-18/IL-12 signaling, which is dominant over the TCR V $\beta$-dependent pathway of MAIT cell activation (Figure 1C). MAIT cell activation also requires MHC-II interaction with SAgs, which can activate conventional $\mathrm{T}$ cells through binding to TCR V $\beta$ chains, and conventional $\mathrm{T}$ cells then promote the production of IL-18 and IL-12 through release of inflammatory mediators $(82,84)$. Upon activation by SAgs, MAIT cells make significant contributions to the cytokine storm via rapid production of proinflammatory cytokines but then are anergized to subsequent bacterial challenge through upregulation of inhibitory receptors such as lymphocyte-activation gene 3, demonstrating that MAIT cells also play a role in pathogenesis in some bacterial infection $(82,83)$.

\section{MAIT CELLS AND DISEASES}

In 2010, two studies reported that MAIT cells reacted to infected cells $(11,65)$. Since that time, there has been a growing body of research describing the role of MAIT cells in disease. Many have suggested that MAIT cells play important roles in infectious diseases, including bacterial and viral diseases, and non-infectious diseases, including autoimmune diseases and cancer; this topic has been reviewed recently $(42,74,85-88)$, so here, we will focus on more recently published articles.

Many studies have described a role of MAIT cells in bacterial infections $(38,89)$. For example, human $\mathrm{CD}^{+}$MAIT cells are important in combating Salmonella enterica serovar Typhi (S. typhi) infection (73) and recently using a mouse model of Salmonella Typhimurium infection, MAIT cells have been shown to accumulate in the lungs of infected mice (37). Similarly, in response to $S$. typhi, MAIT cells are activated and home to affected tissues, such as the gut (73). MAIT cells can also be activated and functionally impaired in patients with scrub typhus, caused by Orientia tsutsugamushi (90).

As mentioned above, MAIT cells may also be involved in the clearance of some viral infections $(45,80)$. In patients with HCV and DENV infections, MAIT cells are present at a lower frequency in blood than in healthy controls, and can be activated in a cytokine-mediated manner to upregulate the expression of IFN- $\gamma$ and $\operatorname{GzmB}(7,78,91)$. Furthermore, there is a negative correlation between the degree of depletion of the intrahepatic MAIT cells and the severity of liver inflammation and fibrosis in $\mathrm{HCV}$-infected patients (78). In IAV infections, human MAIT cells can resist infection by IL-18-dependent activation $(7,66)$. Moreover, the function and frequency of MAIT cells can be impaired and reduced, respectively, in human $\mathrm{T}$ lymphotropic virus type 1 infection (92).

Apart from infectious diseases, MAIT cells also play potential roles in inflammatory diseases and cancers. Patients with ankylosing spondylitis have a lower frequency of MAIT cells in peripheral blood but a higher frequency in synovial fluid (SF) compared with the healthy controls, and MAIT cells in SF of patients with an exaggerated IL-17 phenotype are primed by IL-7 (93). Moreover, human MAIT cells are reduced and functionally altered in the peripheral blood of patients with common variable immunodeficiency (CVID) (94) and primary Sjogren's syndrome (pSS) (68); however, the alteration of MAIT cell function in CVID and pSS patients is different. In CVID patients, MAIT cells are more activated and produce cytokines at higher frequencies than MAIT cells in healthy controls, while in pSS patients, MAIT cells are activated at a lower level and produce less cytokines than MAIT cells in healthy controls. In some cancers, such as gastric, colon, and lung cancers, MAIT cells can migrate from the peripheral blood to affected tissues, express GzmB and perforin, and thus have the potential to kill cancer cells in patients with mucosal-associated cancers (67). Moreover, MAIT cells heavily infiltrate the hepatic metastases in patients with colorectal carcinoma (CRC), and the function of tumor-infiltrating and tumor-margin MAIT cells are impaired irrespective of preoperative chemotherapy, implying the attractiveness of therapeutic targeting of MAIT cells in CRC (95).

However, MAIT cells also play a pathogenic, not protective, role in SAg-mediated illnesses $(82,83)$. MAIT cells activated by SAgs not only contribute to cytokine storm but also acquire an anergic phenotype, indicating a role for them in immunopathology and immunosuppression and also implying that MAIT cells can be used as an efficacious therapeutic target of SAg-mediated illnesses. Collectively, based on an ever increasing understanding of the mechanisms involved in MAIT cell 
activation, MAIT cells continue to be implicated in more and more diseases, often with differing mechanisms of activation.

Given their ability to rapidly produce cytokines after stimulation, direct lysing of microbe-infected cells, and their potential to regulate immune responses, MAIT cells similar to iNKT cells and Vy9V $82 \mathrm{~T}$ cells, which have been used as targets in immunotherapy research (96-101), may also be an effective target for disease treatment. For example, due to their potential role in cancers and infectious diseases, adoptive MAIT cell transfer therapy may be a promising approach to treat diseases (102). Moreover, with a growing number of MAIT cell antigens to be found, the potent antigens can be administered alone or pulsed on APCs to enhance immune responses, or can be used as an effective adjuvant to boost vaccine efficacy. Furthermore, as their pathogenic role in SAg-mediated illnesses, blocking the function of MAIT cells may alleviate the pathological damage caused by SAgs (82). However, there is still no one immunotherapy based on MAIT cells to be used in clinical trials. Therefore, our better understanding of the biology of MAIT cells will accelerate the use of MAIT cell immunotherapy in diseases.

\section{CONCLUSION}

Since the original description in 1993 of a population of unconventional DN T cells with a limited repertoire, much has been learned about the role of MAIT cells in immune response, including a better understanding of MAIT cell phenotypes, restricting molecules, MAIT cell development, antigen recognition, molecular interactions of the TCR-MR1-antigen complex, and their roles in diseases. It was previously believed that in human $\mathrm{T}$ cells expressing $\mathrm{V} \alpha 7.2-\mathrm{J} \alpha 33^{+} \mathrm{TCR} \alpha$ chain were exclusively MAIT cells, but later it was found that, in addition to canonical V $\alpha 7.2-J \alpha 33$ TCR $\alpha$, MAIT cells also express non-canonical $\mathrm{V} \alpha 7.2-\mathrm{J} \alpha 12 / 20$ combinations (29). Similarly, it was originally believed that MR1-restricted T cells were MAIT cells, but later it was confirmed that MR1-restricted T cells are not all MAIT cells, which also contain V $\alpha 7.2^{-}$T cells $(10,61)$. Presently MAIT cells are defined as those T cells which express a V $\alpha 7.2$ TCR $\alpha$ chain and are restricted by MR1. It is now well known that MAIT cell antigens are not only confined to VB2 metabolites. Drugs and drug-related molecules can also serve as MAIT cell agonists (57). MAIT cell activation can also be triggered by SAgs in an MR1independent manner, but dependent on the certain TCR V $\beta$ and/ or cytokine-mediated pathway $(82,83)$.

With more and more antigens being described that are recognized by MAIT cells, along with new and different mechanisms

\section{REFERENCES}

1. Arstila TP, Casrouge A, Baron V, Even J, Kanellopoulos J, Kourilsky P. A direct estimate of the human alphabeta $\mathrm{T}$ cell receptor diversity. Science (1999) 286:958-61. doi:10.1126/science.286.5441.958

2. Godfrey DI, Uldrich AP, McCluskey J, Rossjohn J, Moody DB. The burgeoning family of unconventional T cells. Nat Immunol (2015) 16:1114-23. doi:10.1038/ni.3298

3. Mattner J, Debord KL, Ismail N, Goff RD, Cantu C III, Zhou D, et al. Exogenous and endogenous glycolipid antigens activate NKT cells during microbial infections. Nature (2005) 434:525-9. doi:10.1038/ nature 03408 of activation, our understanding of MAIT cells continues to expand. However, some questions still remain unanswered. Firstly, although the number of exogenous antigens continues to grow, are there endogenous antigens that activate MAIT cells? And what is their nature? Secondly, even though the intracellular trafficking pathway of the MR1-antigen complexes within the cell has been shown $(103,104)$, what intracellular machinery is responsible for retaining empty MR1 molecule in the endoplasmic reticulum to load the extracellular antigens? Thirdly, as MAIT cells are recognized as a potential subset of tissue-resident T cells, what is their contribution to the pathogenesis of tissue or organ (such as liver and lung) diseases? Although some work has described MAIT cells in animals other than human and mouse, what are the roles of MAIT cells play in other animal species? Fourthly, how can MAIT cell responses be modulated, either through small molecules or immunotherapy, to improve human health? Finally, almost all published human data are generated by in vitro assays with a short period of experimental time. Given all the caveats of any in vitro experiments, the findings/observations remain to be verified by in vivo studies in mouse work, albeit the species difference.

Mucosal-associated invariant $\mathrm{T}$ cells, the once obscure cell population, represent up to $10 \%$ of $\mathrm{T}$ cells in the human peripheral blood, therefore, it is imperative that we continue to identify and characterize antigens and mechanisms that activate MAIT cells to better understand the whole scope of the immune response against microorganisms and disease pathogenesis. New insights will lead to improved therapeutics for infectious and non-infectious diseases controlled in part by MAIT cells.

\section{AUTHOR CONTRIBUTIONS}

JC designed the outline, organized the text, and critically revised the manuscript. XX drafted the manuscript. All authors reviewed and approved the final version of the manuscript.

\section{ACKNOWLEDGMENTS}

We thank Dr. Patricia Wilkins at Parasitology Services, USA, for editorial assistance. This work was supported by the Innovative Special Project of Agricultural Sci-Tech (grant no. CAASASTIP-2014-LVRI-09) and Fundamental Research Program of Chinese Academy of Agricultural Sciences (grant no. 0032160017).

4. Kjer-Nielsen L, Patel O, Corbett AJ, Le Nours J, Meehan B, Liu L, et al. MR1 presents microbial vitamin B metabolites to MAIT cells. Nature (2012) 491:717-23. doi:10.1038/nature11605

5. Kurioka A, Ussher JE, Cosgrove C, Clough C, Fergusson JR, Smith K, et al. MAIT cells are licensed through granzyme exchange to kill bacterially sensitized targets. Mucosal Immunol (2015) 8:429-40. doi:10.1038/mi.2014.81

6. Matsuda JL, Mallevaey T, Scott-Browne J, Gapin L. CD1d-restricted iNKT cells, the 'Swiss-Army knife' of the immune system. Curr Opin Immunol (2008) 20:358-68. doi:10.1016/j.coi.2008.03.018

7. van Wilgenburg B, Scherwitzl I, Hutchinson EC, Leng T, Kurioka A, Kulicke C, et al. MAIT cells are activated during human viral infections. Nat Commun (2016) 7:11653. doi:10.1038/ncomms11653 
8. Bendelac A, Bonneville M, Kearney JF. Autoreactivity by design: innate B and T lymphocytes. Nat Rev Immunol (2001) 1:177-86. doi:10.1038/35105052

9. Exley MA. What makes MAITs wait? Immunity (2016) 44:7-9. doi:10.1016/j. immuni.2015.12.021

10. Gherardin NA, Keller AN, Woolley RE, Le Nours J, Ritchie DS, Neeson PJ, et al. Diversity of T cells restricted by the MHC class I-related molecule MR1 facilitates differential antigen recognition. Immunity (2016) 44:32-45. doi:10.1016/j.immuni.2015.12.005

11. Le Bourhis L, Martin E, Peguillet I, Guihot A, Froux N, Core M, et al. Antimicrobial activity of mucosal-associated invariant T cells. Nat Immunol (2010) 11:701-8. doi:10.1038/ni.1890

12. Lantz $\mathrm{O}$, Bendelac $\mathrm{A}$. An invariant $\mathrm{T}$ cell receptor alpha chain is used by a unique subset of major histocompatibility complex class I-specific CD4+ and CD4-8- T cells in mice and humans. J Exp Med (1994) 180:1097-106. doi:10.1084/jem.180.3.1097

13. Dellabona P, Casorati G, Friedli B, Angman L, Sallusto F, Tunnacliffe A, et al. In vivo persistence of expanded clones specific for bacterial antigens within the human T cell receptor alpha/beta CD4-8- subset. J Exp Med (1993) 177:1763-71. doi:10.1084/jem.177.6.1763

14. Dellabona P, Padovan E, Casorati G, Brockhaus M, Lanzavecchia A. An invariant $\mathrm{V}$ alpha $24-\mathrm{J}$ alpha $\mathrm{Q} / \mathrm{V}$ beta $11 \mathrm{~T}$ cell receptor is expressed in all individuals by clonally expanded CD4-8- T cells. J Exp Med (1994) 180:1171-6. doi:10.1084/jem.180.3.1171

15. Porcelli S, Yockey CE, Brenner MB, Balk SP. Analysis of T cell antigen receptor (TCR) expression by human peripheral blood CD4-8-alpha/beta $T$ cells demonstrates preferential use of several $\mathrm{V}$ beta genes and an invariant TCR alpha chain. J Exp Med (1993) 178:1-16. doi:10.1084/jem.178.1.1

16. Bendelac A, Savage PB, Teyton L. The biology of NKT cells. Annu Rev Immunol (2007) 25:297-336. doi:10.1146/annurev.immunol.25.022106.141711

17. Chandra S, Kronenberg M. Activation and function of iNKT and MAIT cells. Adv Immunol (2015) 127:145-201. doi:10.1016/bs.ai.2015.03.003

18. Lynch L, Michelet X, Zhang S, Brennan PJ, Moseman A, Lester C, et al. Regulatory iNKT cells lack expression of the transcription factor PLZF and control the homeostasis of $\mathrm{T}(\mathrm{reg})$ cells and macrophages in adipose tissue. Nat Immunol (2015) 16:85-95. doi:10.1038/ni.3047

19. Kita H, Naidenko OV, Kronenberg M, Ansari AA, Rogers P, He XS, et al. Quantitation and phenotypic analysis of natural killer $\mathrm{T}$ cells in primary biliary cirrhosis using a human CD1d tetramer. Gastroenterology (2002) 123:1031-43. doi:10.1053/gast.2002.36020

20. Lynch L, O'Shea D, Winter DC, Geoghegan J, Doherty DG, O'Farrelly C. Invariant NKT cells and $\mathrm{CD1}(+)$ cells amass in human omentum and are depleted in patients with cancer and obesity. Eur J Immunol (2009) 39:1893-901. doi:10.1002/eji.200939349

21. Gapin L, Godfrey DI, Rossjohn J. Natural Killer T cell obsession with selfantigens. Curr Opin Immunol (2013) 25:168-73. doi:10.1016/j.coi.2013.01.002

22. Kinjo Y, Kitano N, Kronenberg M. The role of invariant natural killer T cells in microbial immunity. J Infect Chemother (2013) 19:560-70. doi:10.1007/ s10156-013-0638-1

23. Kronenberg M, Gapin L. The unconventional lifestyle of NKT cells. Nat Rev Immunol (2002) 2:557-68. doi:10.1038/nri854

24. Mori L, Lepore M, De Libero G. The immunology of CD1- and MR1restricted T cells. Annu Rev Immunol (2016) 34:479-510. doi:10.1146/ annurev-immunol-032414-112008

25. Tilloy F, Treiner E, Park SH, Garcia C, Lemonnier F, de la Salle H, et al. An invariant $\mathrm{T}$ cell receptor alpha chain defines a novel TAP-independent major histocompatibility complex class Ib-restricted alpha/beta $\mathrm{T}$ cell subpopulation in mammals. J Exp Med (1999) 189:1907-21. doi:10.1084/ jem.189.12.1907

26. Treiner E, Duban L, Bahram S, Radosavljevic M, Wanner V, Tilloy F, et al. Selection of evolutionarily conserved mucosal-associated invariant T cells by MR1. Nature (2003) 422:164-9. doi:10.1038/nature01433

27. Riegert P, Wanner V, Bahram S. Genomics, isoforms, expression, and phylogeny of the MHC class I-related MR1 gene. J Immunol (1998) 161:4066-77.

28. Rahimpour A, Koay HF, Enders A, Clanchy R, Eckle SB, Meehan B, et al. Identification of phenotypically and functionally heterogeneous mouse mucosal-associated invariant T cells using MR1 tetramers. J Exp Med (2015) 212:1095-108. doi:10.1084/jem.20142110

29. Reantragoon R, Corbett AJ, Sakala IG, Gherardin NA, Furness JB, Chen Z, et al. Antigen-loaded MR1 tetramers define T cell receptor heterogeneity in mucosal-associated invariant T cells. J Exp Med (2013) 210:2305-20. doi:10.1084/jem.20130958

30. Franciszkiewicz K, Salou M, Legoux F, Zhou Q, Cui Y, Bessoles S, et al. MHC class I-related molecule, MR1, and mucosal-associated invariant T cells. Immunol Rev (2016) 272:120-38. doi:10.1111/imr.12423

31. Walker LJ, Kang YH, Smith MO, Tharmalingham H, Ramamurthy N, Fleming VM, et al. Human MAIT and CD8 $\alpha \alpha$ cells develop from a pool of type-17 precommitted CD8+ T cells. Blood (2012) 119:422-33. doi:10.1182/ blood-2011-05-353789

32. Treiner E, Lantz O. CD1d- and MR1-restricted invariant T cells: of mice and men. Curr Opin Immunol (2006) 18:519-26. doi:10.1016/j.coi.2006.07.001

33. Lepore M, Kalinichenko A, Colone A, Paleja B, Singhal A, Tschumi A, et al. Parallel T-cell cloning and deep sequencing of human MAIT cells reveal stable oligoclonal TCRbeta repertoire. Nat Commun (2014) 5:3866. doi:10.1038/ncomms4866

34. Dias J, Leeansyah E, Sandberg JK. Multiple layers of heterogeneity and subset diversity in human MAIT cell responses to distinct microorganisms and to innate cytokines. Proc Natl Acad Sci U S A (2017) 114:E5434-43. doi:10.1073/pnas.1705759114

35. Martin E, Treiner E, Duban L, Guerri L, Laude H, Toly C, et al. Stepwise development of MAIT cells in mouse and human. PLoS Biol (2009) 7:e54. doi:10.1371/journal.pbio.1000054

36. Koay HF, Gherardin NA, Enders A, Loh L, Mackay LK, Almeida CF, et al. A three-stage intrathymic development pathway for the mucosalassociated invariant $T$ cell lineage. Nat Immunol (2016) 17:1300-11. doi:10.1038/ni.3565

37. Chen Z, Wang H, D'Souza C, Sun S, Kostenko L, Eckle SB, et al. Mucosal-associated invariant T-cell activation and accumulation after in vivo infection depends on microbial riboflavin synthesis and co-stimulatory signals. Mucosal Immunol (2017) 10:58-68. doi:10.1038/mi.2016.39

38. Cui Y, Franciszkiewicz K, Mburu YK, Mondot S, Le Bourhis L, Premel V, et al. Mucosal-associated invariant $\mathrm{T}$ cell-rich congenic mouse strain allows functional evaluation. J Clin Invest (2015) 125:4171-85. doi:10.1172/ JCI82424

39. Leeansyah E, Loh L, Nixon DF, Sandberg JK. Acquisition of innate-like microbial reactivity in mucosal tissues during human fetal MAIT-cell development. Nat Commun (2014) 5:3143. doi:10.1038/ncomms4143

40. Turtle CJ, Delrow J, Joslyn RC, Swanson HM, Basom R, Tabellini L, et al. Innate signals overcome acquired TCR signaling pathway regulation and govern the fate of human CD161(hi) CD8alpha(+) semi-invariant $\mathrm{T}$ cells. Blood (2011) 118:2752-62. doi:10.1182/blood-2011-02-334698

41. Kawachi I, Maldonado J, Strader C, Gilfillan S. MR1-restricted V alpha 19i mucosal-associated invariant $\mathrm{T}$ cells are innate $\mathrm{T}$ cells in the gut lamina propria that provide a rapid and diverse cytokine response. J Immunol (2006) 176:1618-27. doi:10.4049/jimmunol.176.3.1618

42. Le Bourhis L, Mburu YK, Lantz O. MAIT cells, surveyors of a new class of antigen: development and functions. Curr Opin Immunol (2013) 25:174-80. doi:10.1016/j.coi.2013.01.005

43. Sakala IG, Kjer-Nielsen L, Eickhoff CS, Wang X, Blazevic A, Liu L, et al. Functional heterogeneity and antimycobacterial effects of mouse mucosal-associated invariant $\mathrm{T}$ cells specific for riboflavin metabolites. J Immunol (2015) 195:587-601. doi:10.4049/jimmunol.1402545

44. Dusseaux M, Martin E, Serriari N, Peguillet I, Premel V, Louis D, et al. Human MAIT cells are xenobiotic-resistant, tissue-targeted, CD161hi IL-17-secreting T cells. Blood (2011) 117:1250-9. doi:10.1182/blood-201008-303339

45. Ussher JE, Bilton M, Attwod E, Shadwell J, Richardson R, de Lara C, et al. CD161++ CD8+ T cells, including the MAIT cell subset, are specifically activated by IL-12+IL-18 in a TCR-independent manner. Eur J Immunol (2014) 44:195-203. doi:10.1002/eji.201343509

46. Tynan FE, Reid HH, Kjer-Nielsen L, Miles JJ, Wilce MC, Kostenko L, et al. A T cell receptor flattens a bulged antigenic peptide presented by a major histocompatibility complex class I molecule. Nat Immunol (2007) 8:268-76. doi:10.1038/ni1432

47. Van Rhijn I, Kasmar A, de Jong A, Gras S, Bhati M, Doorenspleet ME, et al. A conserved human $\mathrm{T}$ cell population targets mycobacterial antigens presented by CD1b. Nat Immunol (2013) 14:706-13. doi:10.1038/ni.2630

48. Leeansyah E, Ganesh A, Quigley MF, Sonnerborg A, Andersson J, Hunt PW, et al. Activation, exhaustion, and persistent decline of the 
antimicrobial MR1-restricted MAIT-cell population in chronic HIV-1 infection. Blood (2013) 121:1124-35. doi:10.1182/blood-2012-07-445429

49. Croxford JL, Miyake S, Huang YY, Shimamura M, Yamamura T. Invariant $\mathrm{V}$ (alpha)19i $\mathrm{T}$ cells regulate autoimmune inflammation. Nat Immunol (2006) 7:987-94. doi:10.1038/ni1370

50. Corbett AJ, Eckle SB, Birkinshaw RW, Liu L, Patel O, Mahony J, et al. T-cell activation by transitory neo-antigens derived from distinct microbial pathways. Nature (2014) 509:361-5. doi:10.1038/nature13160

51. McWilliam HE, Birkinshaw RW, Villadangos JA, McCluskey J, Rossjohn J. MR1 presentation of vitamin B-based metabolite ligands. Curr Opin Immunol (2015) 34:28-34. doi:10.1016/j.coi.2014.12.004

52. Huang S, Gilfillan S, Kim S, Thompson B, Wang X, Sant AJ, et al. MR1 uses an endocytic pathway to activate mucosal-associated invariant T cells. J Exp Med (2008) 205:1201-11. doi:10.1084/jem.20072579

53. Eckle SB, Birkinshaw RW, Kostenko L, Corbett AJ, McWilliam HE, Reantragoon R, et al. A molecular basis underpinning the T cell receptor heterogeneity of mucosal-associated invariant T cells. J Exp Med (2014) 211:1585-600. doi:10.1084/jem.20140484

54. Mak JY, Xu W, Reid RC, Corbett AJ, Meehan BS, Wang H, et al. Stabilizing short-lived Schiff base derivatives of 5-aminouracils that activate mucosal-associated invariant T cells. Nat Commun (2017) 8:14599. doi:10.1038/ ncomms 14599

55. Soudais C, Samassa F, Sarkis M, Le Bourhis L, Bessoles S, Blanot D, et al. In vitro and in vivo analysis of the Gram-negative bacteria-derived riboflavin precursor derivatives activating mouse MAIT cells. J Immunol (2015) 194:4641-9. doi:10.4049/jimmunol.1403224

56. Eckle SB, Corbett AJ, Keller AN, Chen Z, Godfrey DI, Liu L, et al. Recognition of vitamin B precursors and byproducts by mucosal associated invariant T cells. J Biol Chem (2015) 290:30204-11. doi:10.1074/jbc.R115.685990

57. Keller AN, Eckle SB, Xu W, Liu L, Hughes VA, Mak JY, et al. Drugs and drug-like molecules can modulate the function of mucosal-associated invariant T cells. Nat Immunol (2017) 18:402-11. doi:10.1038/ni.3679

58. Asensi-Fabado MA, Munne-Bosch S. Vitamins in plants: occurrence, biosynthesis and antioxidant function. Trends Plant Sci (2010) 15:582-92. doi:10.1016/j.tplants.2010.07.003

59. Birkinshaw RW, Kjer-Nielsen L, Eckle SB, McCluskey J, Rossjohn J. MAITs, MR1 and vitamin B metabolites. Curr Opin Immunol (2014) 26:7-13. doi:10.1016/j.coi.2013.09.007

60. Gapin L. Check MAIT. JImmunol (2014) 192:4475-80. doi:10.4049/ jimmunol.1400119

61. Meermeier EW, Laugel BF, Sewell AK, Corbett AJ, Rossjohn J, McCluskey J, et al. Human TRAV1-2-negative MR1-restricted T cells detect $S$. pyogenes and alternatives to MAIT riboflavin-based antigens. Nat Commun (2016) 7:12506. doi:10.1038/ncomms12506

62. Lepore M, Kalinichenko A, Calogero S, Kumar P, Paleja B, Schmaler M, et al. Functionally diverse human $\mathrm{T}$ cells recognize non-microbial antigens presented by MR1. Elife (2017) 6:e24476. doi:10.7554/eLife.24476

63. Patel O, Kjer-Nielsen L, Le Nours J, Eckle SB, Birkinshaw R, Beddoe T, et al. Recognition of vitamin B metabolites by mucosal-associated invariant T cells. Nat Commun (2013) 4:2142. doi:10.1038/ncomms3142

64. Reantragoon R, Kjer-Nielsen L, Patel O, Chen Z, Illing PT, Bhati M, et al. Structural insight into MR1-mediated recognition of the mucosal associated invariant T cell receptor. J Exp Med (2012) 209:761-74. doi:10.1084/ jem.20112095

65. Gold MC, Cerri S, Smyk-Pearson S, Cansler ME, Vogt TM, Delepine J, et al. Human mucosal associated invariant $\mathrm{T}$ cells detect bacterially infected cells. PLoS Biol (2010) 8:e1000407. doi:10.1371/journal.pbio.1000407

66. Loh L, Wang Z, Sant S, Koutsakos M, Jegaskanda S, Corbett AJ, et al. Human mucosal-associated invariant $\mathrm{T}$ cells contribute to antiviral influenza immunity via IL-18-dependent activation. Proc Natl Acad Sci U S A (2016) 113:10133-8. doi:10.1073/pnas.1610750113

67. Won EJ, Ju JK, Cho YN, Jin HM, Park KJ, Kim TJ, et al. Clinical relevance of circulating mucosal-associated invariant $\mathrm{T}$ cell levels and their anti-cancer activity in patients with mucosal-associated cancer. Oncotarget (2016) 7:76274-90. doi:10.18632/oncotarget.11187

68. Wang JJ, Macardle C, Weedon H, Beroukas D, Banovic T. Mucosalassociated invariant $\mathrm{T}$ cells are reduced and functionally immature in the peripheral blood of primary Sjogren's syndrome patients. Eur J Immunol (2016) 46:2444-53. doi:10.1002/eji.201646300
69. Chiba A, Tamura N, Yoshikiyo K, Murayama G, Kitagaichi M, Yamaji K, et al. Activation status of mucosal-associated invariant $\mathrm{T}$ cells reflects disease activity and pathology of systemic lupus erythematosus. Arthritis Res Ther (2017) 19:58. doi:10.1186/s13075-017-1257-5

70. Ussher JE, Klenerman P, Willberg CB. Mucosal-associated invariant T-cells: new players in anti-bacterial immunity. Front Immunol (2014) 5:450. doi:10.3389/fimmu.2014.00450

71. Bennett MS, Trivedi S, Iyer AS, Hale JS, Leung DT. Human mucosalassociated invariant $\mathrm{T}$ (MAIT) cells possess capacity for B-cell help. J Leukoc Biol (2017) 102:1261-9. doi:10.1189/jlb.4A0317-116R

72. Meierovics AI, Cowley SC. MAIT cells promote inflammatory monocyte differentiation into dendritic cells during pulmonary intracellular infection. J Exp Med (2016) 213:2793-809. doi:10.1084/jem.20160637

73. Salerno-Goncalves R, Luo D, Fresnay S, Magder L, Darton TC, Jones C, et al. Challenge of humans with wild-type Salmonella enterica serovar Typhi elicits changes in the activation and homing characteristics of mucosalassociated invariant T cells. Front Immunol (2017) 8:398. doi:10.3389/ fimmu.2017.00398

74. Howson LJ, Salio M, Cerundolo V. MR1-restricted mucosal-associated invariant $\mathrm{T}$ cells and their activation during infectious diseases. Front Immunol (2015) 6:303. doi:10.3389/fimmu.2015.00303

75. Sattler A, Dang-Heine C, Reinke P, Babel N. IL-15 dependent induction of IL-18 secretion as a feedback mechanism controlling human MAIT-cell effector functions. Eur J Immunol (2015) 45:2286-98. doi:10.1002/eji. 201445313

76. Le Bourhis L, Dusseaux M, Bohineust A, Bessoles S, Martin E, Premel V, et al. MAIT cells detect and efficiently lyse bacterially-infected epithelial cells. PLoS Pathog (2013) 9:e1003681. doi:10.1371/journal.ppat.1003681

77. Ussher JE, van Wilgenburg B, Hannaway RF, Ruustal K, Phalora P, Kurioka A, et al. TLR signaling in human antigen-presenting cells regulates MR1dependent activation of MAIT cells. Eur J Immunol (2016) 46:1600-14. doi:10.1002/eji.201545969

78. Bolte FJ, O'Keefe AC, Webb LM, Serti E, Rivera E, Liang TJ, et al. Intra-hepatic depletion of mucosal associated invariant $\mathrm{T}$ cells in hepatitis C virus-induced liver inflammation. Gastroenterology (2017) 153:1392-403. doi:10.1053/j.gastro.2017.07.043

79. Jo J, Tan AT, Ussher JE, Sandalova E, Tang XZ, Tan-Garcia A, et al. Tolllike receptor 8 agonist and bacteria trigger potent activation of innate immune cells in human liver. PLoS Pathog (2014) 10:e1004210. doi:10.1371/ journal.ppat. 1004210

80. Chua WJ, Truscott SM, Eickhoff CS, Blazevic A, Hoft DF, Hansen TH. Polyclonal mucosa-associated invariant $\mathrm{T}$ cells have unique innate functions in bacterial infection. Infect Immun (2012) 80:3256-67. doi:10.1128/ IAI.00279-12

81. Jiang J, Chen X, An H, Yang B, Zhang F, Cheng X. Enhanced immune response of MAIT cells in tuberculous pleural effusions depends on cytokine signaling. Sci Rep (2016) 6:32320. doi:10.1038/srep32320

82. Shaler CR, Choi J, Rudak PT, Memarnejadian A, Szabo PA, TunAbraham ME, et al. MAIT cells launch a rapid, robust and distinct hyperinflammatory response to bacterial superantigens and quickly acquire an anergic phenotype that impedes their cognate antimicrobial function: defining a novel mechanism of superantigen-induced immunopathology and immunosuppression. PLoS Biol (2017) 15:e2001930. doi:10.1371/journal. pbio. 2001930

83. Sandberg JK, Norrby-Teglund A, Leeansyah E. Bacterial deception of MAIT cells in a cloud of superantigen and cytokines. PLoS Biol (2017) 15:e2003167. doi:10.1371/journal.pbio.2003167

84. White J, Herman A, Pullen AM, Kubo R, Kappler JW, Marrack P. The $\mathrm{V}$ beta-specific superantigen staphylococcal enterotoxin B: stimulation of mature T cells and clonal deletion in neonatal mice. Cell (1989) 56:27-35. doi:10.1016/0092-8674(89)90980-X

85. Kurioka A, Walker LJ, Klenerman P, Willberg CB. MAIT cells: new guardians of the liver. Clin Transl Immunology (2016) 5:e98. doi:10.1038/cti.2016.51

86. Schwab S, Jobin K, Kurts C. Urinary tract infection: recent insight into the evolutionary arms race between uropathogenic Escherichia coli and our immune system. Nephrol Dial Transplant (2017) 1-7. doi:10.1093/ndt/gfx022

87. Wong EB, Ndung'u T, Kasprowicz VO. The role of mucosal-associated invariant $\mathrm{T}$ cells in infectious diseases. Immunology (2017) 150:45-54. doi:10.1111/ imm.12673 
88. Debusschere K, Lories RJ, Elewaut D. MAIT cells: not just another brick in the wall. Ann Rheum Dis (2016) 75:2057-9. doi:10.1136/annrheumdis2016-209695

89. Meierovics A, Yankelevich WJ, Cowley SC. MAIT cells are critical for optimal mucosal immune responses during in vivo pulmonary bacterial infection. Proc Natl Acad Sci U S A (2013) 110:E3119-28. doi:10.1073/pnas. 1302799110

90. Kang SJ, Jin HM, Won EJ, Cho YN, Jung HJ, Kwon YS, et al. Activation, impaired tumor necrosis factor-alpha production, and deficiency of circulating mucosal-associated invariant $\mathrm{T}$ cells in patients with Scrub typhus. PLoS Negl Trop Dis (2016) 10:e0004832. doi:10.1371/journal.pntd.0004832

91. BeudekerBJB,van OordGW,ArendsJE,SchulzeZurWieschJ,vanderHeideMS, de Knegt RJ, et al. MAIT-cell frequency and function in blood and liver of HCV mono- and HCV/HIV co-infected patients with advanced fibrosis. Liver Int (2017) 1-11. doi:10.1111/liv.13544

92. Paquin-Proulx D, Greenspun BC, Costa EA, Segurado AC, Kallas EG, Nixon DF, et al. MAIT cells are reduced in frequency and functionally impaired in human $\mathrm{T}$ lymphotropic virus type 1 infection: potential clinical implications. PLoS One (2017) 12:e0175345. doi:10.1371/journal. pone. 0175345

93. Gracey E, Qaiyum Z, Almaghlouth I, Lawson D, Karki S, Avvaru N, et al. IL-7 primes IL-17 in mucosal-associated invariant T (MAIT) cells, which contribute to the Th17-axis in ankylosing spondylitis. Ann Rheum Dis (2016) 75:2124-32. doi:10.1136/annrheumdis-2015-208902

94. Arduini S, Dunne J, Conlon N, Feighery C, Doherty DG. Mucosal-associated invariant $\mathrm{T}$ cells are depleted and functionally altered in patients with common variable immunodeficiency. Clin Immunol (2017) 176:23-30. doi:10.1016/j.clim.2016.12.002

95. Shaler CR, Tun-Abraham ME, Skaro AI, Khazaie K, Corbett AJ, Mele T, et al. Mucosa-associated invariant $\mathrm{T}$ cells infiltrate hepatic metastases in patients with colorectal carcinoma but are rendered dysfunctional within and adjacent to tumor microenvironment. Cancer Immunol Immunother (2017). doi:10.1007/s00262-017-2050-7

96. Richter J, Neparidze N, Zhang L, Nair S, Monesmith T, Sundaram R, et al. Clinical regressions and broad immune activation following combination therapy targeting human NKT cells in myeloma. Blood (2013) 121:423-30. doi:10.1182/blood-2012-06-435503

97. Uchida T, Horiguchi S, Tanaka Y, Yamamoto H, Kunii N, Motohashi S, et al. Phase I study of alpha-galactosylceramide-pulsed antigen presenting cells administration to the nasal submucosa in unresectable or recurrent head and neck cancer. Cancer Immunol Immunother (2008) 57:337-45. doi:10.1007/s00262-007-0373-5

98. Bai L, Deng S, Reboulet R, Mathew R, Teyton L, Savage PB, et al. Natural killer T (NKT)-B-cell interactions promote prolonged antibody responses and long-term memory to pneumococcal capsular polysaccharides. Proc Natl Acad Sci U S A (2013) 110:16097-102. doi:10.1073/pnas. 1303218110

99. Kobayashi H, Tanaka Y, Yagi J, Osaka Y, Nakazawa H, Uchiyama T, et al. Safety profile and anti-tumor effects of adoptive immunotherapy using gamma-delta $\mathrm{T}$ cells against advanced renal cell carcinoma: a pilot study. Cancer Immunol Immunother (2007) 56:469-76. doi:10.1007/s00262006-0199-6

100. Salot S, Laplace C, Saiagh S, Bercegeay S, Tenaud I, Cassidanius A, et al. Large scale expansion of gamma 9 delta $2 \mathrm{~T}$ lymphocytes: innacell gamma delta cell therapy product. J Immunol Methods (2007) 326:63-75. doi:10.1016/j.jim.2007.07.010

101. Braza MS, Klein B. Anti-tumour immunotherapy with Vgamma9Vdelta2 T lymphocytes: from the bench to the bedside. Br J Haematol (2013) 160:123-32. doi:10.1111/bjh.12090

102. Guo T, Chamoto K, Hirano N. Adoptive T cell therapy targeting CD1 and MR1. Front Immunol (2015) 6:247. doi:10.3389/fimmu.2015.00247

103. McWilliam HE, Eckle SB, Theodossis A, Liu L, Chen Z, Wubben JM, et al. The intracellular pathway for the presentation of vitamin B-related antigens by the antigen-presenting molecule MR1. Nat Immunol (2016) 17:531-7. doi:10.1038/ni.3416

104. Lamichhane R, Ussher JE. Expression and trafficking of MR1. Immunology (2017) 151:270-9. doi:10.1111/imm.12744

Conflict of Interest Statement: The authors declare that the research was conducted in the absence of any commercial or financial relationships that could be construed as a potential conflict of interest.

Copyright (C) 2017 Xiao and Cai. This is an open-access article distributed under the terms of the Creative Commons Attribution License (CC BY). The use, distribution or reproduction in other forums is permitted, provided the original author(s) or licensor are credited and that the original publication in this journal is cited, in accordance with accepted academic practice. No use, distribution or reproduction is permitted which does not comply with these terms. 